\title{
General Boundary Value Problems for Nonlinear Uniformly Elliptic Equations in Multiply Connected Infinite Domains
}

\author{
Guochun Wen ${ }^{1}$, Yanhui Zhang ${ }^{2}$, Dechang Chen $^{3}$ \\ ${ }^{1}$ School of Mathematical Sciences, Peking University, Beijing, China \\ ${ }^{2}$ Mathematic Department, Beijing Technology and Business University, Beijing, China \\ ${ }^{3}$ Uniformed Services University of the Health Sciences, Bethesda, USA \\ Email: wengc@math.pku.edu.cn, zhangyhchengd@yahoo.com.cn, dechang.chen@usuhs.edu
}

Received May 1, 2013; revised June 29, 2013; accepted July 3, 2013

Copyright (C) 2013 Guochun Wen et al. This is an open access article distributed under the Creative Commons Attribution License, which permits unrestricted use, distribution, and reproduction in any medium, provided the original work is properly cited.

\begin{abstract}
This article discusses the general boundary value problem for the nonlinear uniformly elliptic equation of second order $u_{z \bar{z}}=F\left(z, u, u_{z}, u_{z z}\right)+G\left(z, u, u_{z}\right)$ in $D,(0.1)$ and the boundary condition $\frac{\partial u}{\partial v}+2 c_{1}(z) u=2 c_{2}(z)$ on $\Gamma,(0.2)$ in a multiply connected infinite domain $D$ with the boundary $\Gamma$. The above boundary value problem is called Problem G. Problem G extends the work [8] in which the equation (0.1) includes a nonlinear lower term and the boundary condition $(0.2)$ is more general. If the complex equation (0.1) and the boundary condition (0.2) meet certain assumptions, some solvability results for Problem $\mathrm{G}$ can be obtained. By using reduction to absurdity, we first discuss a priori estimates of solutions and solvability for a modified problem. Then we present results on solvability of Problem G.
\end{abstract}

Keywords: General Boundary Value Problems; Nonlinear Elliptic Equations; Multiply Connected Infinite Domains

\section{Formulation of Elliptic Equations and Boundary Value Problems}

Let $D$ be an $(N+1)$-connected domain which includes the infinite point and has the boundary

$$
\Gamma=\bigcup_{j=0}^{N} \Gamma_{j} \text { in } \mathbb{C} \text {, where } \Gamma \in C_{\mu}^{2}(0<\mu<1) .
$$

Without loss of generality, we assume that $D$ is a circular domain in $|z|>1$, where the boundary consists of $N+1$ circles $\Gamma_{0}=\Gamma_{N+1}=\{|z|=1\}$,

$\Gamma_{j}=\left\{\left|z-z_{j}\right|=r_{j}\right\}, j=1, \cdots, N$ and $z=\infty \in D$. Note that this article uses the same notations as in references [1-8]. We consider the nonlinear uniformly elliptic equation of second order

$$
\left\{\begin{array}{l}
u_{z \bar{z}}=F\left(z, u, u_{z}, u_{z z}\right)+G\left(z, u, u_{z}\right), \\
F=\operatorname{Re}\left[Q u_{z z}+A_{1} u_{z}\right]+A_{2} u+A_{3}, \\
G=G\left(z, u, u_{z}\right), Q=Q\left(z, u, u_{z}, u_{z z}\right), \\
A_{j}=A_{j}\left(z, u, u_{z}\right), j=1,2,3 .
\end{array}\right.
$$

This is the complex form of the nonlinear real equation

$$
\Phi\left(x, y, u, u_{x}, u_{y}, u_{x x}, u_{x y}, u_{y y}\right)=0
$$

with certain conditions (see [3]). We suppose that the Equation (1.1) satisfies Condition C, as described below.

Condition C 1) $Q(z, u, w, U), A_{j}(z, u, w)(j=1,2,3)$ are measurable in $z \in D$ for all continuous functions $u(z), w(z)$ in $\bar{D}$ and all measurable functions $U(z) \in L_{p_{0}, 2}(\bar{D})$, and satisfy

$$
\begin{aligned}
& L_{p, 2}\left[A_{1}(z, u, w), \bar{D}\right] \leq k_{0}, L_{p, 2}\left[A_{2}(z, u, w), \bar{D}\right] \leq \varepsilon k_{0}, \\
& L_{p, 2}\left[A_{3}(z, u, w), \bar{D}\right] \leq k_{1}, A_{2}(z, u, w) \geq 0 \text { in } D,
\end{aligned}
$$

in which $p_{0}, p\left(2<p_{0} \leq p\right), k_{0}, k_{1}, \varepsilon(\leq 1)$ are non-negative constants.

2) The above functions are continuous in $u \in \mathbb{R}, w \in \mathbb{C}$ for almost every $z \in D, U \in \mathbb{C}$, and $Q=0, A_{j}=0(j=1,2,3)$ for $z \notin D$.

3) The Equation (1.1) satisfies the uniform ellipticity condition

$$
\left|F\left(z, u, w, U_{1}\right)-F\left(z, u, w, U_{2}\right)\right| \leq q_{0}\left|U_{1}-U_{2}\right|,
$$

for almost every point $z \in D$, any functions $u(z), w(z) \in C(\bar{D})$ and $U_{1}, U_{2} \in \mathbb{C}$, where $q_{0}(<1)$ 
is a non-negative constant.

4) The function $G(z, u, w)$ possesses the form

$$
G(z, u, w)=B_{1}|w|^{\sigma}+B_{2}|u|^{\tau} \text { in } D
$$

where $u(z), w(z)$ are continuous functions in $\bar{D}$, $0<\sigma, \tau<\infty, L_{p, 2}\left[B_{j}, \bar{D}\right] \leq k_{0}\left(j=1,2,2<p_{0} \leq p\right)$ for a positive constant $k_{0}$.

According to [7], we introduce the general boundary value problem for the Equation (1.1) in $\bar{D}$ as follows.

Problem G Find a continuously differentiable solution $u(z)$ of the second order Equation (1.1) in $\bar{D}$ satisfying the boundary conditions

$$
\begin{aligned}
& \frac{\partial u}{\partial v}+2 c_{1}(z) u=2 c_{2}(z), \\
& \text { i.e. } \operatorname{Re}\left[\overline{\lambda(z)} u_{z}\right]+c_{1}(z) u=c_{2}(z), z \in \Gamma .
\end{aligned}
$$

Here $v$ is a given unit vector at the point $z \in \Gamma$, and $\lambda(z)=\cos (v, x)-i \cos (v, y), \sigma(z)$ and $\tau(z)$ are real functions. We assume $\lambda, c_{1}$ and $c_{2}$ satisfy the conditions

$$
C_{\alpha}[\lambda, \Gamma] \leq k_{0}, C_{\alpha}\left[c_{1}, \Gamma\right] \leq \varepsilon k_{0}, C_{\alpha}\left[c_{2}, \Gamma\right] \leq k_{2},
$$

and

$$
c_{1}(z) \cdot \cos (v, n) \geq 0, z \in \Gamma,
$$

in which $\alpha(1 / 2<\alpha<1), \varepsilon, k_{0}, k_{2}$ are non-negative constant, and $n$ is the unit outer normal at $z \in \Gamma$. If $\cos (v, n)=0, c_{1}(z)=0$ on $\Gamma_{j}, 1 \leq j \leq N$, then we assume that

$$
\int_{\Gamma_{j}} c_{2}(z) \mathrm{d} z=0, u\left(1 / a_{j}^{*}\right)=b_{j}^{*},\left|b_{j}^{*}\right| \leq k_{2}, 1 \leq j \leq N,
$$

in which $a_{j}^{*}$ is a point on $\Gamma_{j}$ and $b_{j}^{*}(j=1, \cdots, N)$ are real constants. There is no harm in assuming that $\cos (v, n)=0, c_{1}(z)=0$ on

$$
\Gamma^{*}=\Gamma_{1} \cup \cdots \cup \Gamma_{N_{0}}\left(N_{0} \leq N\right), \quad \cos (v, n) \text { and } c_{1}(z)
$$

do not both vanish identically on $\Gamma^{* *}=\Gamma_{N_{0}+1} \cup \cdots \cup \Gamma_{N}$.

We can see that the above boundary conditions include some irregular oblique derivative boundary conditions. If $\cos (v, n)>0$ on $\Gamma$, then Problem $G$ is the regular oblique derivative problem (Problem III). If $\cos (v, n)=0$ and $c_{1}=0$ on $\Gamma$, then Problem $G$ is the first boundary value problem, i.e., the Dirichlet boundary value problem (Problem D), in which the boundary condition is

$$
\begin{aligned}
& u(z)=r(z) \\
& =\int_{1 / a_{j}^{*}}^{z} c_{2}(z) \mathrm{d} s+b_{j}^{*}, r\left(1 / a_{j}^{*}\right)=b_{j}^{*}, j=1, \cdots, N+1 .
\end{aligned}
$$

One problem regarding the well posed-ness of Problem G for (1.1) can be formulated as follows:

Problem $\mathbf{H}$ Find a system of continuous functions $[u(z), w(z)]$ of the equation

$$
\left\{\begin{array}{l}
w_{\bar{z}}=F\left(z, u, w, w_{z}\right)+G(z, u, w), \\
F=\operatorname{Re}\left[Q w_{z}+A_{1} w\right]+A_{2} u+A_{3}, \\
G=G(z, u, w), Q=Q\left(z, u, w, w_{z}\right), \\
A_{j}=A_{j}(z, u, w), j=1,2,3, w=u_{z},
\end{array}\right.
$$

satisfying the modified boundary conditions

$$
\begin{aligned}
& \frac{\partial u}{\partial v}+2 c_{1}(z) u=2\left[c_{2}(z)+h(z)\right], \\
& \text { i.e. } \operatorname{Re}\left[\overline{\lambda(z)} u_{z}\right]+c_{1}(z) u=c_{2}(z)+h(z), z \in \Gamma,
\end{aligned}
$$

and the point conditions:

$$
u\left(1 / a_{j}\right)=b_{j}, j=0,1, \cdots, m, a_{0} \in \Gamma_{0}, a_{0} \neq a_{j}(j=1, \cdots, m) .
$$

An explanation of the above conditions is given as follows. The boundary $\Gamma$ can be divided into two parts: $\Gamma^{+} \subset\left\{\cos (v, n) \geq 0, c_{1}(z) \geq 0\right\}$ and

$\Gamma^{-} \subset\left\{\cos (v, n) \leq 0, c_{1}(z) \leq 0\right\}$, such that

$\Gamma^{+} \cup \Gamma^{-}=\Gamma, \Gamma^{+} \cap \Gamma^{-}=\varnothing, \overline{\Gamma^{+}} \cap \overline{\Gamma^{-}}$

$=E=\left\{a_{1}, \cdots, a_{m}, a_{1}^{\prime}, \cdots, a_{l}^{\prime}\right\}$,

every component of

$\Gamma^{+}$and $\Gamma^{-}$includes its initial point, but does not include the terminal point, and there is at least one point on each component of $\Gamma^{+}, \Gamma^{-}$so that $\cos (v, n) \neq 0$. The points $a_{j}(j=1, \cdots, m)$ and $a_{j}^{\prime}(j=1, \cdots, l)$ possess the following property. $a_{j} \in \Gamma^{+}$and $a_{j}^{\prime} \in \Gamma^{-}$, when the direction of $v$ at $a_{j}, a_{j}^{\prime}$ is the same as the direction of $\Gamma$. $a_{j} \in \Gamma^{-}$and $a_{j}^{\prime} \in \Gamma^{+}$, when the direction of $v$ at $a_{j}, a_{j}^{\prime}$ is opposite to the direction of $\Gamma$. And $\cos (v, n)$ changes the sign once on the two components of $\Gamma^{+}, \Gamma^{-}$ with the end point $a_{j}$ or $a_{j}^{\prime}$. And $b_{j}(j=0,1, \cdots, m)$ in (1.12) are real constants satisfying the condition: $\left|b_{j}\right| \leq k_{3}$, herein $k_{3}$ is a non-negative constant. Moreover, the undetermined function $h(z)$ in (1.11) can be written as

$$
h(z)=h_{j} \eta_{j}(z), z \in \Gamma_{j}^{\prime}, j=0,1, \cdots, l .
$$

In (1.13) $\Gamma_{j}^{\prime} \subset \Gamma_{j} \backslash \Gamma^{*}(j=0,1, \cdots, l)$ are non-degenerate, multiply disjointed arcs, each of which consists of inner points of $\Gamma_{j}^{\prime}(j=0,1, \cdots, l)$, such that $\cos (v, n)=0, \sigma(z)=0$ on $\Gamma_{j}^{\prime}(j=1, \cdots, l), a_{0} \in \Gamma_{0}^{\prime}, \Gamma_{0}^{\prime} \cap E=\varnothing$. In addition, $h_{j}(j=0,1, \cdots, l)$ are unknown real constants to be determined appropriately, and $\eta_{j}(z)$ is a positive function on $\Gamma_{j}^{\prime}$ and $\eta_{j}(z)=0$ on $\Gamma \backslash \Gamma_{j}^{\prime}$ and $C_{\alpha}\left[\eta_{j}(z), \Gamma\right] \leq k_{0}, j=0,1, \cdots, l$, in which $\alpha(1 / 2<\alpha<1)$ and $k_{0}$ are non-negative constants. It is not difficult to see that the index of Problem $\mathrm{H}$ is given by

$$
K=\frac{1}{2 \pi} \Delta_{\Gamma} \arg \lambda(z)=N-1+\frac{m-l}{2} .
$$


If $\cos (v, n) \geq 0, c_{1}(z) \geq 0$ on $\Gamma$, then

$\Gamma^{+}=\Gamma, \Gamma^{-}=\varnothing, E=\varnothing$. In this case, Problem H for (1.1) is called Problem O or Problem IV, which includes the Dirichlet problem, the Neumann problem and the regular oblique derivative problem as its special cases. We note that except the case where $\cos (v, n)=0$ and $c_{1}(z)=0$ on $\Gamma$, the conditions (1.12) and (1.13) can be replaced by

$$
\begin{aligned}
& u\left(1 / a_{j}\right)=b_{j}, j=0,1, \cdots, m, \\
& h(z)=h_{j} \eta_{j}(z), z \in \Gamma_{0}^{\prime}, j=1, \cdots, l .
\end{aligned}
$$

with

$$
\left|b_{j}\right| \leq k_{3}, j=0,1, \cdots, m,
$$

in which $k_{3}$ is a non-negative constant. Also note that $[4,7]$ discuss the corresponding problem for the equation (1.1) with $G\left(z, u, u_{z}\right)=0$ in the bounded domains.

\section{A Priori Estimates of Solutions of Boundary Value Problems}

We first give a priori estimates of solutions of Problem $\mathrm{H}$.

Theorem 2.1 Suppose the second order nonlinear Equation (1.10) satisfies Condition C, and $\varepsilon$ in (1.3), (1.7) is small enough. Then any solution

$[u(z), w(z)]=\left[u(z), u_{z}\right]$ of Problem $\mathrm{H}$ for (1.10) with $G(z, u, w)=0$ satisfies the estimates

$$
\begin{gathered}
S(u)=C_{\beta}^{1}[u(z), \bar{D}]+L_{p_{0}, 2}\left[\left|u_{z z}\right|+\left|u_{z \bar{z}}\right|, \bar{D}\right] \leq M_{1}, \\
S(u) \leq M_{2} k_{*}=M_{2}\left(k_{1}+k_{2}+k_{3}\right),
\end{gathered}
$$

in which $\beta=\min \left(\alpha, 1-2 / p_{0}\right), \quad 2<p_{0} \leq p$, $M_{1}=M_{1}\left(q_{0}, p_{0}, k, \alpha, K, D\right), \quad k=\left(k_{1}, k_{2}, k_{3}\right)$, $M_{2}=M_{2}\left(q_{0}, p_{0}, k_{0}, \alpha, K, D\right)$.

Proof First of all, we prove that the solution $u(z)$ of Problem $\mathrm{H}$ satisfies the estimate

$$
S_{1}=C^{1}[u(z), \bar{D}] \leq M_{3}=M_{3}\left(q_{0}, p_{0}, k, \alpha, K, D\right) .
$$

Suppose that the estimate (2.3) is not true. Then there exist sequences of coefficient $\mathrm{s}$ $\left\{Q^{n}\right\},\left\{A_{1}^{n}\right\},\left\{A_{2}^{n}\right\},\left\{A_{3}^{n}\right\},\left\{\lambda_{n}\right\},\left\{c_{1 n}\right\},\left\{c_{2 n}\right\},\left\{b_{j n}^{*}\right\},\left\{b_{j n}\right\}$ of (1.10), (1.11), (1.12) and (1.15) satisfying the same conditions of $Q, A_{1}, A_{2}, A_{3}, \lambda, c_{1}, c_{2}, b_{j}^{*}, b_{j}$, such that $\left\{Q^{n}\right\},\left\{A_{1}^{n}\right\}, \quad\left\{A_{2}^{n}\right\}, \quad\left\{A_{3}^{n}\right\}$ in $D$ weakly converge to $Q^{0}, A_{1}^{0}, A_{2}^{0}, A_{3}^{0}$ respectively, and $\left\{\lambda_{n}\right\},\left\{c_{1 n}\right\},\left\{c_{2 n}\right\},\left\{b_{j n}^{*}\right\}, \quad\left\{b_{j n}\right\}$ on $\Gamma$ uniformly converge to $\lambda_{0}, c_{10}, c_{20}, b_{j 0}^{*}, b_{j 0}$ respectively, and the corresponding boundary value problems

$$
\begin{aligned}
u_{z \bar{z}}-\operatorname{Re}[ & \left.Q^{n} u_{z z}+A_{1}^{n} u_{z}\right]-A_{2}^{n} u=A_{3}^{n}, A_{2}^{n} \geq 0 \text { in } D, \\
& \frac{\partial u}{\partial v_{n}}+2 c_{1 n} u=2 c_{2 n}+2 h_{n}, \\
& c_{1 n}(z) \cdot \cos \left(v_{n}, n\right) \geq 0 \text { on } \Gamma, \int_{\Gamma_{j}} c_{2 n} \mathrm{~d} s=0,
\end{aligned}
$$

$$
\begin{aligned}
& u\left(1 / a_{j}^{*}\right)=b_{j n}^{*}, j=1, \cdots, N_{0}, \\
& u\left(1 / a_{j}\right)=b_{j n}, j=0,1, \cdots, m, n=1,2,
\end{aligned}
$$

have the continuously differentiable solutions $u_{n}(z)(n=1,2, \cdots)$ with the property that $\tilde{H}_{n}=C^{1}\left[u_{n}, \bar{D}\right] \rightarrow \infty$ as $n \rightarrow \infty$. There is no harm in assuming that $\tilde{H}_{n} \geq 1, n=1,2, \cdots$ Denote $U_{n}=u_{n} / \tilde{H}_{n}, n=1,2, \cdots$ It is clear that the function $w_{n}(z)=U_{n z}$ is a solution of the following Riemann-Hilbert boundary value problem

$$
\begin{gathered}
w_{n \bar{z}}-\operatorname{Re}\left[Q^{n} w_{n z}+A_{1}^{n} w_{n}\right]=A^{n}, A^{n}=A_{1}^{n} u_{n}+A_{3}^{n} \text { in } D, \\
u_{n}\left(1 / a_{j}^{*}\right)=b_{j n}^{*}, j=1, \cdots, N_{0}, \\
u_{n}\left(1 / a_{j}\right)=b_{j n}, j=0,1, \cdots, m, n=1,2,
\end{gathered}
$$

where the index of $\lambda_{n}(z)$ is $K=N-1+(m-l) / 2$, and $C\left[w_{n}(z), \bar{D}\right] \leq 1$ showing that $w_{n}(z)$ on $\bar{D}$ is bounded. According to the method in the proof of Theorem 4.7, Chapter I [4], we can obtain that $w_{n}(z)$ satisfies the estimate

$$
L\left(w_{n}\right)=C_{\beta}\left[w_{n}, \bar{D}\right]+L_{p_{0}, 2}\left[\left|w_{n z}\right|+\left|w_{n \bar{z}}\right|, \bar{D}\right] \leq M_{4},
$$

in which $M_{4}=M_{4}\left(q_{0}, p_{0}, k, \alpha, K, D\right)$, and then

$$
U_{n}(z)=-2 \operatorname{Re} \int_{1 / a_{j}^{*}}^{z} \frac{w_{n}(z)}{z^{2}} \mathrm{~d} z+u_{0}(z) / \tilde{H}_{n}
$$

satisfies

$$
S\left(U_{n}\right)=C_{\beta}^{1}\left[U_{n}, \bar{D}\right]+L_{p_{0}, 2}\left[\left|U_{n z z}\right|+\left|U_{n z \bar{z}}\right|, \bar{D}\right] \leq M_{5},
$$

where $M_{5}=M_{5}\left(q_{0}, p_{0}, k, \alpha, K, D\right)$. Hence from $\left\{U_{n}(z)\right\}$ and $\left\{U_{n z}\right\}$, we can choose the subsequences $\left\{U_{n_{k}}(z)\right\}$ and $\left\{U_{n_{k} z}\right\}$, which uniformly converge to $U_{0}(z)$ and $U_{0 z}$ in $\bar{D}$ respectively, such that $U_{0}(z)$ is a solution of the following boundary value problem

$$
\begin{gathered}
U_{z \bar{z}}-\operatorname{Re}\left[Q^{0} U_{z z}+A_{1}^{0} U_{z}\right]-A_{2}^{0} U=0, A_{2}^{0} \geq 0 \text { in } D, \quad \text { (2.11) } \\
\frac{\partial U}{\partial v_{0}}+2 c_{10} u=2 h_{0}, c_{10}(z) \cdot \cos \left(v_{0}, n\right) \geq 0 \text { on } \Gamma, \quad \text { (2.12) } \\
U\left(1 / a_{j}^{*}\right)=0, j=1, \cdots, N_{0}, U\left(1 / a_{j}\right)=0, j=0,1, \cdots, m .
\end{gathered}
$$

By the uniqueness of solutions of Problem $\mathrm{H}$ (see Theorem 2.3 below), we see that $U(z)=0$ on $\bar{D}$. However from $C^{1}\left[U_{n}(z), \bar{D}\right]=1$, it can be derived that $C^{1}\left[U_{0}(z), \bar{D}\right]=1$. This contradiction proves that (2.3) is true. Afterwards, using the method of deriving (2.9) from $C^{1}\left[U_{n}, \bar{D}\right]=1$, we can obtain the estimate (2.1). The estimate (2.2) can be concluded from (2.1).

Theorem 2.2 Let the Equation (1.1) satisfy Condition 
C and $\varepsilon$ in (1.3), (1.7) be a sufficiently small positive constant. Then any solution $[w(z), u(z)]$ of Problem $\mathrm{H}$ for (1.10) satisfies the estimates

$$
\begin{array}{r}
C_{\beta}[w(z), \bar{D}]+C_{\beta}[u(z), \bar{D}] \leq M_{6} k_{*}, \\
L_{p_{0}, 2}\left[\left|w_{\bar{z}}\right|+\left|w_{z}\right|, \bar{D}\right]+L_{p_{0}, 2}\left[u_{z}, \bar{D}\right] \leq M_{7} k_{*},
\end{array}
$$

where $\beta, p_{0}$ are as stated in Theorem 2.1, $M_{j}=M_{j}\left(q_{0}, p_{0}, k_{0}, \alpha, K, D\right), j=6,7$,

$$
k_{*}=k_{1}+k_{2}+k_{3}+k_{0}\left\{[C(w, \bar{D})]^{\sigma}+[C(u, \bar{D})]^{\tau}\right\} .
$$

Proof It is easy to see that $[w(z), u(z)]$ of Problem $\mathrm{H}$ for (1.10) satisfies the following equation and boun- dary conditions:

$$
\begin{array}{r}
w_{\bar{z}}-\operatorname{Re}\left[Q w_{z}\right]+A_{1} w=A_{2} u+A_{3}+G, z \in D, \\
\operatorname{Re}[\overline{\lambda(z)} w(z)]=-c_{1} u+c_{2}(z)+h(z), z \in \Gamma, \\
u\left(1 / a_{j}^{*}\right)=b_{j n}^{*}, j=1, \cdots, N_{0}, \\
u\left(1 / a_{j}\right)=b_{j n}, j=0,1, \cdots, m, n=1,2,
\end{array}
$$

By using the same method as in the proof of Theorem 2.1, we can obtain the estimates (2.14) and (2.15).

Now we discuss the uniqueness of solutions of Problem $\mathrm{H}$ for the nonlinear elliptic Equation (1.1) with $G(z, u, w)=0$. For this, we need to consider the following condition

$$
\left\{\begin{array}{l}
F\left(z, u_{1}, u_{1 z}, U\right)-F\left(z, u_{2}, u_{2 z}, U\right)=\operatorname{Re}\left[\tilde{A}_{1}\left(u_{1}-u_{2}\right)_{z}\right]+\tilde{A}_{2}\left(u_{1}-u_{2}\right), \\
\tilde{A}_{j}=\tilde{A}_{j}\left(z, u_{1}, u_{2}, U\right), j=1,2, L_{p_{0}, 2}\left[\tilde{A}_{j}, \bar{D}\right] \leq k_{0}, 2<p_{0} \leq p
\end{array}\right.
$$

for any continuously differentiable functions $u_{j}(z) \in C_{\beta}^{1}(\bar{D}), j=1,2$ and any measurable function $U(z) \in L_{p_{0}, 2}(\bar{D})$, where $\beta=\left[\min \left(\alpha, 1-2 / p_{0}\right)\right]$, $p_{0}\left(2<p_{0} \leq p\right), \quad k_{0}$ are constants as stated in Section 1 . We can prove the uniqueness of solutions of Problem $\mathrm{H}$ for (1.1).

Theorem 2.3 Let the second order nonlinear Equation (1.1) satisfy Condition C and (2.19) with $\tilde{A}_{2} \geq 0$ in $D$. Then the solution of Problem $\mathrm{H}$ for (1.10) with $G\left(z, u, u_{z}\right)=0$ is unique.

Proof Let $u_{1}(z), u_{2}(z)$ be two solutions of Problem
$\mathrm{H}$ for (1.10). By the above conditions, we see that $u(z)=u_{1}(z)-u_{2}(z)$ is a solution of the following boundary value problem Problem

$$
\begin{array}{r}
u_{z \bar{z}}-\operatorname{Re}\left[\tilde{Q} u_{z z}+\tilde{A}_{1} u_{z}\right]-\tilde{A}_{2} u=0, z \in D, \quad(2.20) \\
\frac{\partial u}{\partial v}+2 c_{1}(z) u(z)=2 H(z), z \in \Gamma, \quad(2.21) \\
u\left(1 / a_{j}^{*}\right)=0, j=1, \cdots, N_{0}, u\left(1 / a_{j}\right)=0, h=0,1, \cdots, m,
\end{array}
$$

with

$$
\left\{\begin{array}{l}
\operatorname{Re}\left[\tilde{Q}\left(u_{1}-u_{2}\right)_{z z}\right]=F\left(z, u_{1}, u_{1 z}, u_{1 z z}\right)-F\left(z, u_{1}, u_{1 z}, u_{2 z z}\right), \\
\operatorname{Re}\left[\tilde{A}_{1}\left(u_{1}-u_{2}\right)_{z}\right]=F\left(z, u_{1}, u_{1 z}, u_{2 z z}\right)-F\left(z, u_{1}, u_{2 z}, u_{2 z z}\right), \\
\tilde{A}_{2}= \begin{cases}\frac{F\left(z, u_{1}, u_{2 z}, u_{2 z z}\right)-F\left(z, u_{2}, u_{2 z}, u_{2 z z}\right)}{u_{1}-u_{2}} & \text { for } u_{1}(z) \neq u_{2}(z), \\
0 & \text { for } u_{1}(z)=u_{2}(z), z \in D,\end{cases} \\
|\tilde{Q}| \leq q_{0}<1, L_{p_{0}, 2}\left[\tilde{A}_{j}, \bar{D}\right]<\infty, j=1,2, \tilde{A}_{2} \geq 0 \text { in } D,
\end{array}\right.
$$

where $q_{0}, p_{0}, k_{1}$ are non-negative constants. According to the proof of Theorem 2.6, Chapter I, [4], and using the extremum principle of solutions for (2.20) (see Chapter 3, [3]), we can prove that $u(z)=0$ in $D$, and then $u_{1}(z)=u_{2}(z)$ in $D$.

\section{Solvability of Boundary Value Problems}

We first prove a lemma.

Lemma 3.1. If $G(z, u, w)$ satisfies the condition stated in Condition $\mathrm{C}$, then the nonlinear mapping $T$ : $C(\bar{D}) \times C(\bar{D}) \rightarrow L_{p, 2}(\bar{D})$ defined by

$$
\begin{gathered}
G=G[z, u(z), w(z)] \text { is coninuous and bounded with } \\
L_{p, 2}[G(z, u(z), w(z)), \bar{D}] \leq L_{p, 2}\left[B_{1}, \bar{D}\right] \\
{[C(w, \bar{D})]^{\sigma}+L_{p, 2}\left[B_{2}, \bar{D}\right][C(u, \bar{D})]^{\tau}}
\end{gathered}
$$

where $p=p_{0}>2$.

Proof In order to prove that the mapping $T$ :

$C(\bar{D}) \times C(\bar{D}) \rightarrow L_{p, 2}(\bar{D})$ defined by

$G=G[z, u(z), w(z)]$ is continuous, we choose any sequence of functions

$\left[w_{n}(z), u_{n}(z)\right]\left(w_{n}(z), u_{n}(z) \in C(\bar{D}), n=0,1,2, \cdots\right)$ 
such that $C\left[w_{n}-w_{0}, \bar{D}\right]+C\left[u_{n}-u_{0}, \bar{D}\right] \rightarrow 0$ as $n \rightarrow \infty$. Similarly to Lemma 2.2.1 [5], we can prove that $C_{n}=G\left(z, u_{n}, w_{n}\right)-G\left(z, u_{0}, w_{0}\right)$ possesses the property that

$$
L_{p, 2}\left[C_{n}, \bar{D}\right] \rightarrow 0 \text { as } n \rightarrow \infty .
$$

And the inequality (3.1) is obviously true.

Theorem 3.2. Let the complex Equation (1.1) satisfy Condition $\mathrm{C}$, and the positive constant $\varepsilon$ in (1.3) and (1.7) be small enough.

1) When $0<\sigma, \tau<1$, Problem $\mathrm{H}$ for the Equation (1.10) has a solution $[w(z), u(z)]$, where $w(z), u(z) \in W_{p_{0}, 2}^{1}(D)$ with the constant

$p_{0}\left(2<p_{0} \leq p\right)$ as stated before.

2) When $\min (\sigma, \tau)>1$, Problem $H$ for (1.10) has a solution $[w(z), u(z)]$, where $w(z) \in W_{p_{0}, 2}^{1}(D)$, provided that

$$
M_{8}=L_{p_{0}, 2}\left[A_{3}, \bar{D}\right]+C_{\alpha}\left[c_{2}, \Gamma\right]+\sum_{j=0}^{m}\left|b_{j}\right|
$$

is sufficiently small.

Proof 1) In this case, the algebraic equation for $t$ becomes

$$
M_{9}\left\{L_{p, 2}\left[A_{3}, \bar{D}\right]+L_{p, 2}\left[B_{1}, \bar{D}\right] t^{\sigma}+L_{p, 2}\left[B_{2}, \bar{D}\right] t^{\tau}+L_{\alpha}\left[C_{2}, \Gamma\right]+\sum_{j=0}^{m}\left|b_{j}\right|\right\}=t,
$$

with $M_{9}=M_{6}+M_{7}$, where $M_{6}, M_{7}$ are constants as stated in (2.14) and (2.15). Because $0<\sigma, \tau<1$, Equation (3.4) has a unique solution $t=M_{10}>0$. Now we introduce a bounded, closed and convex subset $B^{*}$ of the Banach space $C(\bar{D}) \times C(\bar{D})$, whose elements are of the form $[w(z), u(z)]$ satisfying the condition

$$
w(z), u(z) \in C(\bar{D}), C[w(z), \bar{D}]+C[u(z), \bar{D}] \leq M_{10} .
$$

We choose a pair of functions $[\tilde{w}(z), \tilde{u}(z)] \in B^{*}$ and substitute it into the appropriate positions of

$F\left(z, u, w, w_{z}\right), G(z, u, w)$ in (1.10) and the boundary condition (1.11) to obtain

$$
\begin{array}{r}
w_{\bar{z}}=\tilde{F}\left(z, u, w, \tilde{u}, \tilde{w}, w_{z}\right)+G(z, \tilde{u}, \tilde{w}), \\
\operatorname{Re}[\overline{\lambda(z)} w(z)]=-c_{1}(z) \tilde{u}+c_{2}(z), z \in \Gamma,
\end{array}
$$

$$
\begin{aligned}
\tilde{F} & \left(z, u, w, \tilde{u}, \tilde{w}, w_{z}\right) \\
\text { where }= & \operatorname{Re}\left[Q\left(z, \tilde{u}, \tilde{w}, w_{z}\right) w_{z}+A_{1}(z, \tilde{u}, \tilde{w}) w\right] \\
& +A_{2}(z, \tilde{u}, \tilde{w}) u+A_{3}(z, \tilde{u}, \tilde{w}) .
\end{aligned}
$$

In accordance with the method in the proof of Theorem 1.2.5 [5], we can prove that the boundary value problem (3.6), (3.7) and (1.15) has a unique solution $[w(z), u(z)]$. Denote by $[w, u]=T[\tilde{w}(z), \tilde{u}(z)]$ the mapping from $[\tilde{w}(z), \tilde{u}(z)]$ to $[w(z), u(z)]$. Noting that

$$
L_{p, 2}\left[A_{2} u, \bar{D}\right] \leq \varepsilon M_{10} k_{0}, C_{\alpha}\left[-C_{1} u, \Gamma\right] \leq \varepsilon M_{10} k_{0},
$$

provided that the positive number $\varepsilon$ is sufficiently small, and noting that the coefficients of complex Equation (3.6) satisfy the same conditions as in Condition C, from Theorem 2.2, we can obtain

$$
\begin{aligned}
& C[w, \bar{D}]+L_{p_{0}, 2}\left[\left|w_{\bar{z}}\right|+\left|w_{z}\right|, \bar{D}\right]+C[u, \bar{D}]+L_{p_{0}, 2}\left[u_{z}, \bar{D}\right] \leq M_{9}\left\{L_{p, 2}\left[A_{3}, \bar{D}\right]+C_{\alpha}\left[c_{2}, \Gamma\right]+\sum_{j=0}^{m}\left|b_{j}\right|+L_{p, 2}[G, \bar{D}]\right\} \\
& \leq M_{9}\left\{M_{8}+L_{p, 2}\left[B_{1}, \bar{D}\right] C[\tilde{w}, \bar{D}]^{\sigma}+L_{p, 2}\left[B_{2}, \bar{D}\right] C[\tilde{u}, \bar{D}]^{\tau}\right\} \leq M_{9}\left\{M_{8}+L_{p, 2}\left[B_{1}, \bar{D}\right] M_{10}^{\sigma}+L_{p, 2}\left[B_{2}, \bar{D}\right] M_{10}^{\tau}\right\}=M_{10} .
\end{aligned}
$$

This shows that $T$ maps $B^{*}$ onto a compact subset in $B^{*}$. Next, we verify that $T$ in $B^{*}$ is a continuous operator. In fact, we arbitrarily select a sequence $\left\{\tilde{w}_{n}(z), \tilde{u}_{n}(z)\right\}$ in $B^{*}$, such that

$$
C\left(\tilde{w}_{n}-\tilde{w}_{0}, \bar{D}\right)+C\left(\tilde{u}_{n}-\tilde{u}_{0}, \bar{D}\right) \rightarrow 0 \text { as } n \rightarrow \infty .
$$

By Lemma 3.1, we can see that

$$
\begin{aligned}
& L_{p, 2}\left[A_{j}\left(z, \tilde{u}_{n}, \tilde{w}_{n}\right)-A_{j}\left(z, \tilde{u}_{0}, \tilde{w}_{0}\right), \bar{D}\right] \\
& \quad \rightarrow 0(j=1,2,3) \text { as } n \rightarrow \infty .
\end{aligned}
$$

Moreover, from $\left[w_{n}, u_{n}\right]=T\left[\tilde{w}_{n}, \tilde{u}_{n}\right],\left[w_{0}, u_{0}\right]=T\left[\tilde{w}_{0}, \tilde{u}_{0}\right]$, it is clear that $\left[w_{n}-w_{0}, u_{n}-u_{0}\right]$ is a solution of Problem $\mathrm{H}$ for the following equation:

$$
\begin{aligned}
\left(w_{n}-w_{0}\right)_{\bar{z}}= & \tilde{F}\left(z, u_{n}, w_{n}, \tilde{u}_{n}, \tilde{w}_{n}, w_{n z}\right) \\
& -\tilde{F}\left(z, u_{0}, w_{0}, \tilde{u}_{0}, \tilde{w}_{0}, w_{0 z}\right) \\
& +G\left(z, \tilde{u}_{n}, \tilde{w}_{n}\right)-G\left(z, \tilde{u}_{0}, \tilde{w}_{0}\right) \text { in } D, \\
& \operatorname{Re}\left[\overline{\lambda(z)}\left(w_{n}-w_{0}\right)\right] \\
& =-c_{1}(z)\left(\tilde{u}_{n}-\tilde{u}_{0}\right)+h(z) \text { on } \Gamma,
\end{aligned}
$$




$$
\begin{aligned}
& u_{n}\left(1 / a_{j}^{*}\right)-u_{0}\left(1 / a_{j}^{*}\right)=0, j=1, \cdots, N_{0}, \\
& u_{n}\left(1 / a_{j}\right)-u_{0}\left(1 / a_{j}\right)=0, j=0,1, \cdots, m .
\end{aligned}
$$

In accordance with the method in proof of Theorem 2.2, we can obtain the estimate

$$
\begin{aligned}
C & {\left[w_{n}-w_{0}, \bar{D}\right]+L_{p_{0}, 2}\left[\left|\left(w_{n}-w_{0}\right)_{\bar{z}}\right|+\left|\left(w_{n}-w_{0}\right)_{z}\right|, \bar{D}\right] } \\
+ & C\left[u_{n}-u_{0}, \bar{D}\right]+L_{p_{0}, 2}\left[\left(u_{n}-u_{0}\right)_{z}, \bar{D}\right] \\
\leq & M_{11}\left\{\varepsilon L_{p, 2}\left[A_{2}\left(z, \tilde{u}_{n}, \tilde{w}_{n}\right) \tilde{u}_{n}-A_{2}\left(z, \tilde{u}_{0}, \tilde{w}_{0}\right) \tilde{u}_{0}, \bar{D}\right]\right. \\
& +L_{p, 2}\left[A_{3}\left(z, \tilde{u}_{n}, \tilde{w}_{n}\right)-A_{3}\left(z, \tilde{u}_{0}, \tilde{w}_{0}\right), \bar{D}\right] \\
& \left.+L_{p, 2}\left[G\left(z, \tilde{u}_{n}, \tilde{w}_{n}\right)-G\left(z, \tilde{u}_{0}, \tilde{w}_{0}\right), \bar{D}\right]+\varepsilon C_{\alpha}\left[C_{1}(z)\left(\tilde{u}_{n}-\tilde{u}_{0}\right), \Gamma\right]\right\},
\end{aligned}
$$

in which $M_{11}=M_{11}\left(q_{0}, p_{0}, k_{0}, \alpha, K, D\right)$. From (3.9), (3.10) and the above estimate, we obtain

$C\left[w_{n}-w_{0}, \bar{D}\right]+C\left[u_{n}-u_{0}, \bar{D}\right] \rightarrow 0$ as $n \rightarrow \infty$. On the basis of the Schauder fixed-point theorem, there exists a function $[w(z), u(z)](w(z), u(z) \in C(\bar{D}))$ such that $[w(z), u(z)]=T[w(z), u(z)]$. And from Theorem 2.2, it is easy to see that $w(z), u(z) \in W_{p_{0}, 2}^{1}(D)$, and $[w(z), u(z)]$ is a solution of Problem $\mathrm{H}$ for the Equation (1.10) with the condition $0<\sigma, \tau<1$.

In addition, using a method similar to the above, we see that if $G(z, u, w)=\operatorname{Re} B_{1} w+B_{2}|u|^{\tau}$ in $D$, where $0<\tau<1, L_{p, 2}\left[B_{j}, \bar{D}\right] \leq k_{0}<\infty, j=1,2$, then the above solvability result still holds.

2) Secondly, we discuss the case, where $\min (\sigma, \tau)>1$. In this case, (3.4) has the solution $t=M_{10}$ provided that $M_{8}$ in (3.3) is small enough. We consider a closed and convex subset $B_{*}$ in the Banach space $C(\bar{D}) \times C(\bar{D})$, i.e.,

$$
B_{*}=\left\{w(z), u(z) \in C(\bar{D}), C[w, \bar{D}]+C[u, \bar{D}] \leq M_{10}\right\} .
$$

Applying a similar method as before, we can verify that there exists a solution

$[w(z), u(z)] \in W_{p_{0}, 2}^{1}(D) \times W_{p_{0}, 2}^{1}(D)$ of Problem $\mathrm{H}$ for (1.10) with the condition $\min (\sigma, \tau)>1$.

Moreover, if $G(z, u, w)=\operatorname{Re} B_{1} w+B_{2}|u|^{\tau} \quad$ in $D$, where $1<\tau<\infty, \quad L_{p, 2}\left[B_{j}, \bar{D}\right] \leq k_{0}<\infty, j=1,2$, then under the same condition, we can derive the above solvability result by a similar method.

From the above theorem, the next result can be derived.

Theorem 3.3 Under the same conditions as in Theorem 3.2, Problem G has $l+1$ solvability conditions, and the general solution $u(z)$ includes $m+1$ arbitrary real constants.

Proof Let the solution $[w(z), u(z)]$ of Problem $\mathrm{H}$ for (1.10) be substituted into the boundary condition (1.11). If the function $h(z)=0, z \in \Gamma$, i.e.

$h_{j}=0, z \in \Gamma^{\prime}, j=0,1, \cdots, l$, then we have $w(z)=u_{z}$ in $D$ and the function $u(z)$ is just a solution of Problem
$\mathrm{G}$ for (1.1). Hence the total number $l+1$ of above equalities is just the number of solvability conditions of Problem G.

Also note that the real constants $b_{j}(j=0,1, \cdots, m)$ in (1.12) and (1.15) are arbitrarily chosen. This shows that the general solution of Problem G for (1.1) includes the $m+1$ arbitrary real constants as stated in the theorem.

Note: The opinions expressed herein are those of the authors and do not necessarily represent those of the Uniformed Services University of the Health Sciences and the Department of Defense.

\section{REFERENCES}

[1] I. N. Vekua, “Generalized Analytic Functions,” Pergamon, Oxford, 1962.

[2] G. C. Wen, "Linear and Nonlinear Elliptic Complex Equations,” Shanghai Scientific and Technical Publishers, Shanghai, 1986. (in Chinese)

[3] G. C. Wen, "Conformal Mappings and Boundary Value Problems,” American Mathematical Society, Providence, 1992.

[4] G. C. Wen, "Approximate Methods and Numerical Analysis for Elliptic Complex Equations,” Gordon and Breach, Amsterdam, 1999.

[5] G. C. Wen, D. C. Chen and Z. L. Xu, "Nonlinear Complex Analysis and its Applications, Mathematics Monograph Series 12,” Science Press, Beijing, 2008.

[6] G. C. Wen, "Recent Progress in Theory and Applications of Modern Complex Analysis,” Science Press, Beijing, 2010.

[7] G. C. Wen and C. C. Yang, "On General Boundary Value Problems for Nonlinear Elliptic Equations of Second Order in a Multiply Connected Domain,” Acta Applicandae Mathematicae, Vol. 43 No. 2, 1996, pp. 169-189. doi:10.1007/BF00047923

[8] G. C. Wen, "Irregular Oblique Derivative Problems for Second Order Nonlinear Elliptic Equations on Infinite Domains," Electronic Journal of Differential Equations, Vol. 2012, No. 142, 2012, pp. 1-8. 